\title{
Absolute Stability of a Class of Trilateral Haptic Systems
}

\author{
Jian Li, Student Member, IEEE, Mahdi Tavakoli, Member, IEEE, and Qi Huang, Senior Member, IEEE
}

\begin{abstract}
Trilateral haptic systems can be modeled as three-port networks. We present a criterion for absolute stability of a general class of three-port networks. Traditionally, existing (i.e., Llewellyn's) criteria have facilitated the stability analysis of bilateral haptic systems modeled as two-port networks. If the same criteria were to be used for stability analysis of a three-port network, its third port termination would need to be assumed known for it to reduce to a two-port network. This is restrictive because, for absolute stability, all three terminations of the three-port network must be allowed to be arbitrary (while passive). Extending Llewellyn's criterion, we present closed-form necessary and sufficient conditions for absolute stability of a general class of three-port networks. We first find a symmetrization condition under which a general asymmetric impedance (or admittance) matrix $Z_{3 \times 3}$ has a symmetric equivalent $Z_{e q}$ from a network stability perspective. Then, via the equivalence of passivity and absolute stability for reciprocal networks, an absolute stability condition for the original nonreciprocal network is derived. To demonstrate the convenience and utility of using this criterion for both analysis and design, it is applied to the problem of designing stabilizing controllers for dual-user haptic teleoperation systems, with simulations and experiments validating the criterion.
\end{abstract}

Index Terms-Three-port network, trilateral haptic system, absolute stability.

\section{INTRODUCTION}

A Bilateral master-slave teleoperation system can be modeled as a two-port network [1]. For coupled stability analysis of such a system, the knowledge of the human operator's and the environment's dynamics is needed in addition to the teleoperation system's immittance ( $z$ and $y$ ) parameters. In practice, however, the model for the human operator and the environment can be unknown, uncertain, and/or time-varying. Thus, absolute or unconditional stability of a bilateral haptic system based on the assumption that the human operator and the environment demonstrate passive behaviors is analyzed via Llewellyn's stability criterion for two-port networks [2], [3], [4]. For brevity, absolute or unconditional stability is simply referred to as "stability" in the rest of the paper. "Coupled stability" will refer to BIBO stability of a network when it is coupled to terminations at all if its ports.

Recently, new application scenarios have emerged that involve the collaboration of multiple users in teleoperation of a robot or in performing a haptic virtual task. Examples of these new applications are

- Jian Li is with the School of Energy Science and Engineering, University of Electronic Science and Technology of China, Chengdu, Sichuan, 611731 China.

- Jian Li and Mahdi Tavakoli are with the Department of Electrical and Computer Engineering, University of Alberta, Edmonton, Alberta, T6G 2V4 Canada.

- Qi Huang is with the School of Energy Science and Engineering, University of Electronic Science and Technology of China, Chengdu, Sichuan, 611731 China.

E-mail: uajian@gmail.com,_mahdi.tavakoli@ualberta.ca, huangqi@uestc.edu.cn. tele-rehabilitation [5], surgical training [6], and cooperative multi-robot systems [7]. Specifically, dual-user teleoperation of a robot and triple-user collaborative haptic virtual environments have given rise to trilateral haptic systems. A difference between a trilateral and a bilateral haptic system is that they are modeled as a three-port and a two-port network, respectively. Thus, conventional theories for stability analysis of bilateral haptic systems will not be adequate for trilateral haptic systems.

In contrast to the stability criteria for two-port networks, which have only involved conditions on the immittance parameters of the two-port network and are independent of the port terminations, past research has been struggling to find a similar stability condition for three-port networks independent of the port terminations. Instead, in past research [8], [9], [10], the third port was assumed to be coupled to a known termination such that the three-port network reduced to a two-port network, paving the way for the application of Llewellyn's criterion. The limiting factor of this approach is that the resulting stability condition will inevitably depend on the immittance of the third port's termination. This is restrictive because not allowing all three terminations of the three-port network to be arbitrary (while passive) contradicts the very definition of stability (again, throughout this paper, all references are to absolute or unconditional stability).

Using the aforementioned approach, namely, reducing a given three-port network to a two-port network by assuming a known termination for the third port, Boehm et al. in [11] established nine conditions for determining the stability of a three-port network de- 
scribed by its scattering $(S)$ parameters. The approach in [9] reduced a three-port network to three two-port networks by terminating each of the three ports, and managed to reduce the number of conditions from nine to three. Also, Kuo et al. [8] reduced a three-port network to a two-port network by coupling the third port to a known termination and then required the input reflection coefficients at the first and the second ports to be less than unity. Unfortunately, in the above approaches, a degree of freedom is lost when the third port is coupled to a known termination. Thus, there is a need for a tool that can directly analyze the stability of trilateral haptic systems modeled as three-port networks without reducing them to two-port networks. Such a tool, which will guarantee the coupled stability of the system under all passive but otherwise arbitrary linear time-invariant (LTI) terminations for all three ports, is developed in this paper.

Unlike past work, we would like to have a stability condition directly in the immittance (e.g., impedance $Z$ ) domain and not in the scattering $(S)$ domain. While the $S$-parameters are most accurately measured for higher-frequency systems such as microwave circuits, $Z$-parameters can be accurately measured in lowerfrequency systems including robotic systems. In fact, the measurement of $Z$-parameters approaches zero in microwave circuits where the frequencies are very high (over $1 \mathrm{GHz}$ ), making the use of reflection coefficients and scattering parameters justifiable for the stability analysis. This explains the abundance of scattering parameters based stability conditions in the microwave systems literature (see, for example, [12]). Conversely, in robotic systems, the measurement of $S$ parameters is close to zero in any frequency range of practical interest and, therefore, it is highly desirable to have stability conditions that directly depend on the $Z$-parameters or other immittance parameters of the three-port network.

Inspired by $\mathrm{Ku}$ [13], who studied $N$-port network stability when the impedance matrix of the network is of a tri-diagonal Jacobian form [13], in this paper we present a criterion to analyze the stability of a general class of nonreciprocal three-port networks. As a case study, we consider a trilateral haptic system for dual-user collaborative teleoperation [10], [14] and use the proposed stability criterion to design stabilizing controllers for the system.

The rest of the paper is organized as follows: The next section reviews definitions of stability for general $N$-port networks and, for the special case of reciprocal networks, relates them to passivity. In Section 3, the proposed stability criterion for all nonreciprocal three-port networks that satisfy our so-called symmetrization condition is derived. Then, as a case study to show how the resulting stability criterion can be utilized, in Section 4, a trilateral shared control architecture for dual-user collaborative teleoperation systems is considered and the stability conditions in terms of system parameters including controller gains are found. Finally, simulations and experiments to verify the validity of the calculated stability conditions for the position-position dual-user teleoperation system are presented in Section 5. Section 6 contains concluding remarks and future work.

\section{Definitions and Criteria for N-Port Network Stability}

Consider an LTI system with impulse response $h(t)$. The system's transfer function is the Laplace transform of $h(t)$ defined as

$$
H(s)=\int_{0}^{\infty} h(t) e^{-s t} d t
$$

where $s=\sigma+j \omega . H(s)$ is stable if every bounded input produces a bounded output and this happens if the poles of $H(s)$ have negative real parts. This stability definition is equivalent to the absolute convergence of $H(s)$ in the region $\operatorname{Re}(s) \geq 0 . H(s)$ is said to converge absolutely if the integral $\int_{0}^{\infty}\left|h(t) e^{-s t}\right| d t$ exists. The set of values of $s$ for which $H(s)$ converges is known as the region of convergence (ROC) and is of the form $\operatorname{Re}(s) \geq a$, where $a$ is a real constant. Importantly, if $H(s)$ converges at $s=s_{0}$, then it automatically converges for all $s$ with $\operatorname{Re}(s)>\operatorname{Re}\left(s_{0}\right)$. The above means that for stability analysis it suffices to focus on the convergence of $H(s)$ when $\operatorname{Re}(s)=0$, i.e., on the $j \omega$ axis.

An $n$-port network is called stable if the steady-state port currents are zero under passive LTI terminations for all ports [15]. Similarly, an $n$-port network is called weakly stable if the steady-state port currents are zero under strictly passive LTI terminations for all ports. We know that an LTI termination is passive (strictly passive) if its impedance is nonnegative (positive) real [16]. Suppose the $n$-port network is terminated in arbitrary passive impedances $z_{1}(j \omega), z_{2}(j \omega), \cdots, z_{n}(j \omega)$, and the port currents at the respective ports are denoted by $I_{1}, I_{2}, \cdots, I_{n}$. Thus, it is immediately understood that a general $n$-port network with impedance matrix $Z_{n \times n}$ is stable (weakly stable) if and only if, for $s=j \omega$, the equation

$$
\left(Z(s)+Z_{0}(s)\right) \mathbf{I}=0, \quad Z_{0}(s)=\operatorname{diag}\left[z_{1}, z_{2}, \cdots, z_{n}\right]
$$

where $\mathbf{I}=\left[I_{1}, I_{2}, \cdots, I_{n}\right]^{T}$ has only the trivial solution $\mathbf{I}=0$ for every choice of $n$ terminations $z_{1}, z_{2}, \cdots, z_{n}$ that are nonnegative (positive) real. In other words, the $n$-port network is stable (weakly stable) if and only if

$$
\operatorname{det}\left(Z(s)+Z_{0}(s)\right) \neq 0
$$

for $s=j \omega$ and for any choice of $n$ LTI terminations with nonnegative (positive) real parts. We remember 
that a nonnegative (positive) real impedance $z_{i}(j \omega)$ has a real part with a nonnegative (positive) value ${ }^{1}$.

There is an alternate definition for $n$-port network stability. Assume the input impedance (i.e., the driving-point impedances) at port $\kappa$ of an $n$-port network is $Z_{\kappa}$ when all other $n-1$ ports are coupled to passive (strictly passive) terminations. Then, the $n$ port network is stable (weakly stable) if and only if

$$
\operatorname{Re}\left(Z_{\kappa}(s)\right)>0 \quad(\geq 0), \quad \kappa=1,2, \cdots, n
$$

for $s=j \omega$ and for all passive (strictly passive) $z_{1}$, $z_{2}, \cdots, z_{n}$. Equivalently, the $n$-port network is stable (weakly stable) if and only if

$$
\int_{0}^{t} V_{\kappa}(\tau) I_{\kappa}(\tau) \mathrm{d} \tau>0 \quad(\geq 0), \quad \kappa=1,2, \cdots, n
$$

for all passive (strictly passive) $z_{1}, z_{2}, \cdots, z_{n}$ [17]. Conditions (4) or (5) represent an alternate way to examine the stability of $n$-port networks. In the following, the equivalence of (4) and (5) with the stability condition (3) is shown for $n=3$, i.e., a general nonreciprocal three-port network shown in Figure 1(a) with the impedance matrix

$$
Z=\left[\begin{array}{lll}
Z_{11} & Z_{12} & Z_{13} \\
Z_{21} & Z_{22} & Z_{23} \\
Z_{31} & Z_{32} & Z_{33}
\end{array}\right]
$$

For the three-port network (6), the input impedances at port 1 when ports 2 and 3 are terminated to $z_{2}$ and $z_{3}$, at port 2 when ports 1 and 3 are terminated to $z_{1}$ and $z_{3}$, and at port 3 when ports 1 and 2 are terminated to $z_{1}$ and $z_{2}$ are, respectively,

$$
Z_{1}=Z_{11}-\frac{Z_{12} Z_{21}\left(Z_{33}+z_{3}\right)+Z_{13} Z_{31}\left(Z_{22}+z_{2}\right)-D}{\left(Z_{22}+z_{2}\right)\left(Z_{33}+z_{3}\right)-Z_{23} Z_{32}}
$$

$Z_{2}=Z_{22}-\frac{Z_{12} Z_{21}\left(Z_{33}+z_{3}\right)+Z_{23} Z_{32}\left(Z_{11}+z_{1}\right)-D}{\left(Z_{11}+z_{1}\right)\left(Z_{33}+z_{3}\right)-Z_{13} Z_{31}}$

$Z_{3}=Z_{33}-\frac{Z_{13} Z_{31}\left(Z_{22}+z_{2}\right)+Z_{23} Z_{32}\left(Z_{11}+z_{1}\right)-D}{\left(Z_{11}+z_{1}\right)\left(Z_{22}+z_{2}\right)-Z_{12} Z_{21}}$

where $D=Z_{13} Z_{21} Z_{32}-Z_{12} Z_{23} Z_{31}$. According to (3), for the nonreciprocal three-port network (6) to be stable (weakly stable), we must have

$$
\operatorname{det}\left(Z(s)+Z_{0}(s)\right) \neq 0, \quad Z_{0}(s)=\operatorname{diag}\left[z_{1}, z_{2}, z_{3}\right]
$$

for $s=j \omega$, and for any choice of passive (strictly passive) impedances $z_{1}, z_{2}$ and $z_{3}$. The stability condition (10) can be rewritten as

$$
\left(Z_{11}+z_{1}\right)\left(Z_{22}+z_{2}\right)\left(Z_{33}+z_{3}\right)-Z_{13} Z_{21} Z_{32}-Z_{12} Z_{23} Z_{31}
$$$$
\neq Z_{23} Z_{32}\left(Z_{11}+z_{1}\right)+Z_{12} Z_{21}\left(Z_{33}+z_{3}\right)
$$$$
+Z_{13} Z_{31}\left(Z_{22}+z_{2}\right)
$$

1. A rational function $F(s)$ is positive real if and only if, in addition to being real for real $s, F(s)$ has no RHP poles, any poles of $F(s)$ on the imaginary axis are simple with real and non-negative residues, and $\operatorname{Re}[F(j \omega)] \geq 0, \forall \omega$.
Now, if $\left(Z_{22}+z_{2}\right)\left(Z_{33}+z_{3}\right)-Z_{23} Z_{32} \neq 0$, (11) implies that

$-z_{1} \neq Z_{11}-\frac{Z_{12} Z_{21}\left(Z_{33}+z_{3}\right)+Z_{13} Z_{31}\left(Z_{22}+z_{2}\right)-D}{\left(Z_{22}+z_{2}\right)\left(Z_{33}+z_{3}\right)-Z_{23} Z_{32}}$

Likewise, we get similar inequality conditions for $-z_{2}$ and $-z_{3}$. So, the stability condition (10) is satisfied if and only if

$$
-z_{\kappa}(s) \neq Z_{\kappa}(s), \quad \kappa=1,2,3
$$

for $s=j \omega$. Now, recall that the real part of $z_{\kappa}$ covers the closed right half plane (open right half plane) if it is passive (strictly passive). Thus, the three-port network is stable (weakly stable) if and only if

$$
\operatorname{Re}\left(Z_{\kappa}(s)\right)>0 \quad(\geq 0), \quad \kappa=1,2,3
$$

for all $s=j \omega$, and for all passive (strictly passive) $z_{1}$, $z_{2}$ and $z_{3}$.

\section{Main Result: A Stability Criterion FOR A ClASS OF NONRECIPROCAL THREE- PORT NETWORKS}

The previous stability definitions can hardly be used as closed-form stability criteria for general nonreciprocal networks. Instead, we will introduce an approach in this section that utilizes Lemma 1 below for checking the stability of a reciprocal network, which has a symmetric impedance matrix. Also, Lemma 2 will be used for finding the symmetric equivalent of an asymmetric impedance matrix from a network stability perspective. Lastly, Lemma 3 and Lemma 4 will be required in the proof of Theorem 1 .

Lemma 1. [15] Let $Z=Z^{\mathrm{T}}$ be the impedance matrix of a reciprocal n-port network. Then, the network is passive (strictly passive), i.e., $\operatorname{Re} Z \geq 0(\operatorname{Re} Z>0)$, if and only if it is weakly stable (stable).

Lemma 2. [18] Let $Z_{1}$ and $Z_{2}$ be the impedance matrices of two n-port networks. Then, if $Z_{1}$ and $Z_{2}$ possess identical principal minors of all orders, the two n-port networks are stable (weakly stable) together.

In fact, [18] showed that if $Z_{1}$ and $Z_{2}$ have identical principal minors of all orders, then

$$
\operatorname{det}\left(Z_{1}+Z_{0}\right)=\operatorname{det}\left(Z_{2}+Z_{0}\right)
$$

Therefore, the stability (weak stability) of the two networks with impedance matrices $Z_{1}$ and $Z_{2}$ will happen at the same time because of (3), which is to hold for all passive (strictly passive) $Z_{0}=$ $\operatorname{diag}\left[z_{1}, z_{2}, \cdots, z_{n}\right]$.

Lemma 3. [19] A symmetric matrix is positive definite (positive semi-definite) if and only if the determinants of every principal minor is positive (nonnegative).

Lemma 4. [20] If the determinant of the real parts of the elements of a symmetrical non-singular complex matrix 
$Z$ is positive (nonnegative), then the determinant of the real parts of the elements of the $Z^{-1}$ is also positive (nonnegative).

Now, we propose the following theorem as a compact, straightforward, and easy-to-check condition for the stability of a general nonreciprocal three-port network.

Theorem 1. The nonreciprocal three-port network with the impedance matrix $Z$ in (6) satisfying the symmetrization condition

$$
Z_{13} Z_{21} Z_{32}-Z_{12} Z_{23} Z_{31}=0
$$

is stable (weakly stable) if and only if

$\begin{array}{ll}\operatorname{Re}\left(Z_{11}\right)>0 & (\geq 0), \\ \operatorname{Re}\left(Z_{22}\right)>0 \quad & (\geq 0), \\ \operatorname{Re}\left(Z_{33}\right)>0 \quad & (\geq 0),\end{array}$

$\operatorname{Re}\left(Z_{11}\right) \operatorname{Re}\left(Z_{22}\right)-\frac{\left|Z_{12} Z_{21}\right|+\operatorname{Re}\left(Z_{12} Z_{21}\right)}{2}>0 \quad(\geq 0)$,

$\operatorname{Re}\left(Z_{11}\right) \operatorname{Re}\left(Z_{33}\right)-\frac{\left|Z_{13} Z_{31}\right|+\operatorname{Re}\left(Z_{13} Z_{31}\right)}{2}>0 \quad(\geq 0)$,

$\operatorname{Re}\left(Z_{22}\right) \operatorname{Re}\left(Z_{33}\right)-\frac{\left|Z_{23} Z_{32}\right|+\operatorname{Re}\left(Z_{23} Z_{32}\right)}{2}>0 \quad(\geq 0)$,

and

$$
\begin{aligned}
& \operatorname{Re}\left(Z_{11}\right) \operatorname{Re}\left(Z_{22}\right) \operatorname{Re}\left(Z_{33}\right) \\
& -\operatorname{Re}\left(Z_{11}\right) \frac{\left|Z_{23} Z_{32}\right|+\operatorname{Re}\left(Z_{23} Z_{32}\right)}{2} \\
& -\operatorname{Re}\left(Z_{22}\right) \frac{\left|Z_{13} Z_{31}\right|+\operatorname{Re}\left(Z_{13} Z_{31}\right)}{2} \\
& -\operatorname{Re}\left(Z_{33}\right) \frac{\left|Z_{12} Z_{21}\right|+\operatorname{Re}\left(Z_{12} Z_{21}\right)}{2} \\
& +2 \operatorname{Re}\left(\sqrt{Z_{12} Z_{21}}\right) \operatorname{Re}\left(\sqrt{Z_{13} Z_{31}}\right) \operatorname{Re}\left(\sqrt{Z_{23} Z_{32}}\right)>0
\end{aligned}
$$

Proof: According to Lemma 2, if there exists a reciprocal three-port network with impedance matrix $Z_{e q}$ that has the same stability (weak stability) characterization as the nonreciprocal three-port network with impedance matrix $Z$, then

$$
\operatorname{det}\left(Z_{e q}+Z_{0}\right)=\operatorname{det}\left(Z+Z_{0}\right)
$$

for any passive (strictly passive) $Z_{0}=\operatorname{diag}\left[z_{1}, z_{2}, z_{3}\right]$. Now, if and only if the symmetrization condition (16) holds, solving (18) for $Z_{e q}$ given $Z$ in (6) gives the following independent of $Z_{0}$ :

$Z_{e q}=\left[\begin{array}{ccc}Z_{11} & \gamma_{1} \sqrt{Z_{12} Z_{21}} & \gamma_{2} \sqrt{Z_{13} Z_{31}} \\ \gamma_{1} \sqrt{Z_{12} Z_{21}} & Z_{22} & \gamma_{3} \sqrt{Z_{23} Z_{32}} \\ \gamma_{2} \sqrt{Z_{13} Z_{31}} & \gamma_{3} \sqrt{Z_{23} Z_{32}} & Z_{33}\end{array}\right]$

where $\gamma_{i}= \pm 1$ for $i=1,2,3$. We will discuss later why the stability condition will be the same for any of these 8 solutions.
According to Lemma 1, the symmetric three-port network with the impedance matrix $Z_{e q}$ given in (19) is stable (weakly stable) if and only if it is strictly passive (passive), i.e.,

$$
\operatorname{Re}\left(Z_{e q}\right)>0 \quad(\geq 0)
$$

Consequently, if (16) holds, then the nonreciprocal three-port network with the impedance matrix $Z$ given in (6) is stable (weakly stable) if and only if the matrix $\operatorname{Re}\left(Z_{e q}\right)$, with $Z_{e q}$ given in (19), is positive definite (positive semi-definite). After simplifying the matrix $\operatorname{Re}\left(Z_{e q}\right)$ by

$$
\begin{aligned}
& \left(\operatorname{Re}\left(\sqrt{Z_{i j} Z_{j i}}\right)\right)=\sqrt{\frac{\left|Z_{i j} Z_{j i}\right|+R e\left(Z_{i j} Z_{j i}\right)}{2}} \\
& \quad i, j=1,2,3
\end{aligned}
$$

and using Lemma 3, we arrive at conditions (17a)-(17g) for positive definiteness (positive semidefiniteness) of $\operatorname{Re}\left(Z_{e q}\right)$. Note that any of the 8 choices caused by taking $\gamma_{i}= \pm 1, i=1,2,3$, in (19) will result in the same stability conditions (17a)-(17g) due to the fact that we are calculating the determinants of the principal minors of $Z_{e q}$. This concludes the proof.

Remark 1. Note that Theorem 1 holds not only for the impedance matrix (6) of a general nonreciprocal network but also for its admittance matrix. The reason for this is Lemma 3 and Lemma 4. In fact, the positive definiteness (positive semi-definiteness) of the equivalent reciprocal network is independent of whether an impedance representation or an admittance representation is used for it.

Remark 2. For the special case of $Z_{13}=Z_{23}=$ $Z_{31}=Z_{32}=Z_{33}=0$, Theorem 1 simplifies to the stability criterion for nonreciprocal two-port networks best known as Llewellyn's criterion [2], [3], [4], [20]. Also, for the special case of $Z_{13}=Z_{31}=0$, Theorem 1 simplifies to the stability criterion in [13]. Our Theorem 1 is more general as it lifts those constraints and is applicable to any nonreciprocal three-port network whose impedance matrix $Z$ satisfies the symmetrization condition (16). As we will demonstrate in the next section, the symmetrization condition (16) is a limitation but it is mild and can be fulfilled by appropriate choice of free parameters in the three-port network (e.g., controller structure, authority sharing laws, and gains in the case of trilateral haptic systems).

Remark 3. For four-port networks, we can use a similar procedure as that outlined in this section. We find that a nonreciprocal four-port network with impedance matrix $Z_{4 \times 4}$ can be converted to a reciprocal four-port network with the same stability characteristics if and only if $Z_{23} Z_{34} Z_{42}=Z_{24} Z_{32} Z_{43}$, $Z_{13} Z_{21} Z_{32}=Z_{12} Z_{23} Z_{31}, Z_{14} Z_{21} Z_{42}=Z_{12} Z_{24} Z_{41}$, and $Z_{14} Z_{31} Z_{43}=Z_{13} Z_{34} Z_{41}$. We will not pursue four-port stability analysis because of the significant complexity associated with this symmetrization condition set and because our current focus is trilateral haptic systems. 


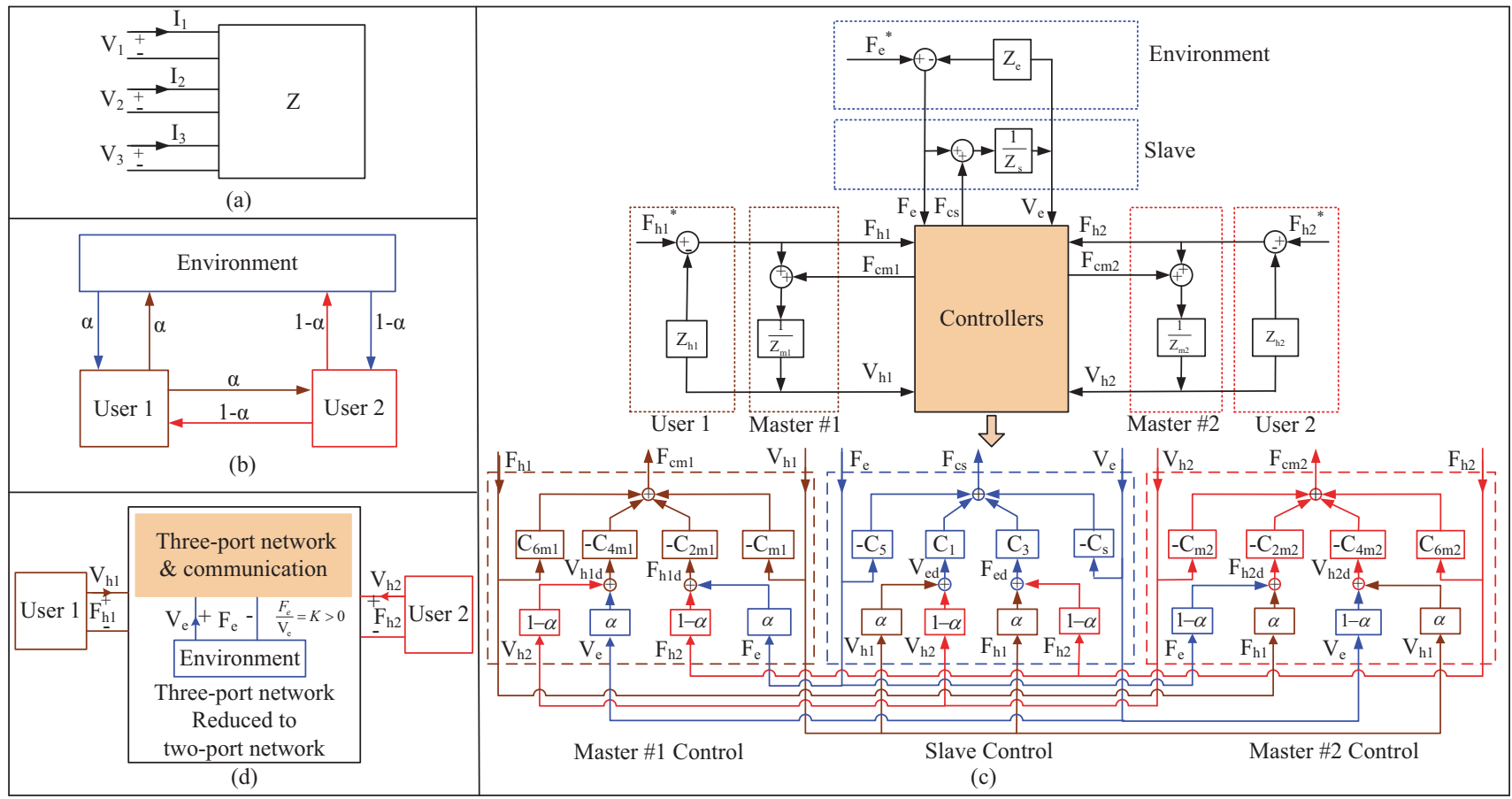

Fig. 1. (a) A general three-port network. (b) Dual-user haptic teleoperation. (c) A dual-user haptic teleoperation system under four-channel control. (d) Reduced two-port network between user 1 and user 2.

\section{Case Study: Application of the Pro- posed Criterion to Trilateral haptic SYSTEMS}

In this section, the aim is to apply the proposed stability criterion, which is general and can be used for three-port networks in various applications, to a trilateral haptic system. A trilateral haptic system may be a collaborative haptic virtual environment with three users, or a dual-user haptic teleoperation system with one slave robot. In the following, for brevity, we only consider the latter and a similar procedure case may be followed for the former. We begin by reviewing a four-channel, dual-user teleoperation system and specifically investigate the stability of positionposition and force-position control schemes.

\subsection{A Four-channel Dual-user Shared Control Teleoperation System}

In a dual-user teleoperation control system, the goal is that two users collaboratively control a robot. Such a system consists of two master robots as haptic interfaces for the two users and one slave robot to perform a desired task on an environment. This finds application in many real-world scenarios such as when the aim is to train a novice trainee (user 1) to do a task under haptic guidance from a mentor (user 2). As elaborated by [6], [10], the reference position and force for each robot are sums of positions and forces of the other two robots weighted by a parameter $\alpha \in[0,1]$ that specifies their relative control authorities - see Figure 1(b). Therefore, $\alpha$ affects how the trainee and the mentor collaborate and contribute to the reference position for the slave and what share of force feedback each of them receives. For instance, if $\alpha=0$, the slave robot will be completely controlled by the mentor and the trainee will receive large force feedback urging $\mathrm{him} /$ her to follow the mentor's motions. On the other hand, if $\alpha=1$, the slave robot is completely controlled by the trainee, allowing the mentor to assess the skill level of the trainee by feeling the reflected forces. If $0<\alpha<1$, the trainee and the mentor collaborate and each contribute to the slave robot position while receiving some force feedback.

Consider the dual-user teleoperation system shown in Figure 1(c). The dynamics of the two masters and the slave in contact with the two users and the environment, respectively, are

$$
\begin{aligned}
Z_{m i} V_{h i} & =F_{h i}+F_{c m i} \\
Z_{s} V_{e} & =F_{e}+F_{c s}
\end{aligned}
$$

where $i=1,2$, and $Z_{m i}$ and $Z_{s}$ are the impedances of the two masters and the slave, respectively. Also, $F_{h i}$ denotes the interaction force between each user and the corresponding master and $F_{s}$ denotes the interaction force between the slave and the environment. Lastly, $V_{h i}$ and $V_{e}$ are the users and the environment velocities.

The four-channel dual-user shared control laws in Figure 1(c) are [10], [21], [14]:

$$
\begin{gathered}
F_{c m i}=-C_{m i} V_{h i}-C_{4 m i} V_{h i d}+C_{6 m i} F_{h i}-C_{2 m i} F_{h i d} \\
F_{c s}=-C_{s} V_{e}+C_{1} V_{e d}-C_{5} F_{e}+C_{3} F_{e d}
\end{gathered}
$$


where $C_{m i}$ and $C_{s}$ are local position controllers, $C_{6 m i}$ and $C_{5}$ are local force controllers, and $C_{1}, C_{2 m i}$, $C_{3}$, and $C_{4 m i}$ are feedforward and feedback compensators. Also, $V_{h i d}$ and $V_{e d}$ are the reference velocities and $F_{h i d}$ and $F_{e d}$ are the references forces for the two masters and the slave, where using the complementary-linear-combination (CLC) laws for authority sharing are

$$
\begin{aligned}
V_{h 1 d} & =\alpha V_{e}+(1-\alpha) V_{h 2} \\
V_{h 2 d} & =(1-\alpha) V_{e}+\alpha V_{h 1} \\
V_{e d} & =\alpha V_{h 1}+(1-\alpha) V_{h 2} \\
F_{h 1 d} & =\alpha F_{e}+(1-\alpha) F_{h 2} \\
F_{h 2 d} & =(1-\alpha) F_{e}+\alpha F_{h 1} \\
F_{e d} & =\alpha F_{h 1}+(1-\alpha) F_{h 2}
\end{aligned}
$$

It is easy to verify that the reference velocities (positions) and references forces in (24) are consistent with the trainee/mentor collaboration scenario discussed above.

By substituting (24) in (23) and then substituting the result in (22), the impedance matrix representation of the closed-loop dual-user teleoperation system is found as

$$
\left[\begin{array}{c}
F_{h 1} \\
F_{h 2} \\
F_{e}
\end{array}\right]=\left[\begin{array}{lll}
Z_{11} & Z_{12} & Z_{13} \\
Z_{21} & Z_{22} & Z_{23} \\
Z_{31} & Z_{32} & Z_{33}
\end{array}\right]\left[\begin{array}{c}
V_{h 1} \\
V_{h 2} \\
V_{e}
\end{array}\right]
$$

where $Z=A^{-1} B$ with

$$
\begin{gathered}
A=\left[\begin{array}{ccc}
C_{6 m 1}+1 & -(1-\alpha) C_{2 m 1} & -\alpha C_{2 m 1} \\
-\alpha C_{2 m 2} & C_{6 m 2}+1 & -(1-\alpha) C_{2 m 2} \\
\alpha C_{3} & (1-\alpha) C_{3} & 1+C_{5}
\end{array}\right] \\
B=\left[\begin{array}{ccc}
C_{m 1}+Z_{m 1} & (1-\alpha) C_{4 m 1} & \alpha C_{4 m 1} \\
\alpha C_{4 m 2} & C_{m 2}+Z_{m 2} & (1-\alpha) C_{4 m 2} \\
-\alpha C_{1} & -(1-\alpha) C_{1} & C_{s}+Z_{s}
\end{array}\right]
\end{gathered}
$$

In the next subsections, we will consider two special cases of the above, namely position-position and force-position shared control architectures [22] and analyze their stability.

\subsection{Position-Position Dual-user Teleoperation}

Position-position control is a special case of fourchannel control in which there is no need for any force sensor measurements. In this control architecture, we have $C_{2 m 1}=C_{2 m 2}=C_{3}=C_{5}=C_{6 m 1}=C_{6 m 2}=0$. For good position tracking, the common choice is $C_{1}=C_{s}, C_{4 m 1}=-C_{m 1}$, and $C_{4 m 2}=-C_{m 2}$. Then, the impedance matrix of dual-user teleoperation system can be found from (25) but is not shown here.

In the following two subsections, we will discuss two methods to design a stable position-position controlled dual-user haptic teleoperation system. The first method tries to find an equivalent bilateral teleoperation system for the trilateral teleoperation system by coupling one port to a known termination and then utilizes Llewellyn's criterion for finding the stability conditions. The second method is based on Theorem 1 for direct stability analysis of a three-port network for three passive but otherwise arbitrary terminations. We will show that the latter approach is better.

\subsubsection{Stability Analysis via Reduction to Two-Port Networks}

To reduce a three-port network to an equivalent twoport network between the two users (Figure 1(d)), one can couple the environment port to a known load termination and then absorb the load termination into the network. To find the equivalent twoport impedance matrix, in the simplest case, one can consider the aforementioned load to be a pure known stiffness $K>0$. Assume $\alpha=\frac{1}{2}, Z_{m 1}=M_{m 1} s$, $Z_{m 2}=M_{m 2} s, Z_{s}=M_{s} s$, and let us make the following choices for the controllers:

$$
\begin{aligned}
C_{m 1} & =\frac{K_{p m 1}+K_{v m 1} s}{s}, \quad C_{m 2}=\frac{K_{p m 2}+K_{v m 2} s}{s}, \\
C_{s} & =\frac{K_{p s}+K_{v s} s}{s}
\end{aligned}
$$

Then, using $\frac{F_{e}}{V_{e}}=K$, the equivalent two-port network for the dual-user teleoperation control system is given by

$$
\left[\begin{array}{l}
F_{h 1} \\
F_{h 2}
\end{array}\right]=\left[\begin{array}{ll}
Z_{11}^{\prime} & Z_{12}^{\prime} \\
Z_{21}^{\prime} & Z_{22}^{\prime}
\end{array}\right]\left[\begin{array}{l}
V_{h 1} \\
V_{h 2}
\end{array}\right]
$$

For brevity, we do not show the elements of the matrix $Z^{\prime}(j \omega)$.

Now, the stability of the reduced two-port network (27) must be tested for all possible choices of $K$ and all frequencies $\omega$. By Llewellyn's criterion, the stability of the dual-user teleoperation system is guaranteed if, for all $K$ and all $\omega$, we have

$$
\begin{aligned}
& K_{v m 1}-\frac{1}{4} \frac{\left(K_{v s} K_{v m 1} \omega^{2}-K_{p s} K_{p m 1}\right)\left(K+K_{v s}\right)}{\left(K+K_{v s}\right)^{2} \omega^{2}+\left(K_{p s}-M_{s} \omega^{2}\right)^{2}} \\
& -\frac{1}{4} \frac{\left.\left(K_{v s} K_{p m 1}+K_{v m 1} K_{p s}\right)\left(K_{p s}-M_{s} \omega^{2}\right)\right)}{\left(K+K_{v s}\right)^{2} \omega^{2}+\left(K_{p s}-M_{s} \omega^{2}\right)^{2}} \geq 0 \\
& \frac{1}{4} \frac{\left(K_{p m 2}+K_{v m 2}\right)\left(K_{p s} K+K_{v s} M_{s} \omega^{2}\right)}{\left(K+K_{v s}\right)^{2} \omega^{2}+\left(K_{p s}-M_{s} \omega^{2}\right)^{2}} \geq 0 \\
& 2 \operatorname{Re}\left(Z_{11}^{\prime}\right) \operatorname{Re}\left(Z_{22}^{\prime}\right)-\operatorname{Re}\left(Z_{12}^{\prime} Z_{21}^{\prime}\right)-\left|Z_{12}^{\prime} Z_{21}^{\prime}\right| \geq 0
\end{aligned}
$$

To synthesize controllers based on (28) for all values of $K$ and $\omega$ is a daunting task if not impossible. This issue is exacerbated once one considers that the environment port's load may include damping and inertia in addition to stiffness, in which case (28) would have to be satisfied for all values ranging from 0 to $\infty$ of stiffness, damping, inertia and frequency. As discussed in [10], the computational burden can be alleviated by using the transformation $\Gamma=\frac{Z_{e}-1}{Z_{e}+1}$, where $Z_{e}$ is the complex impedance of the load termination, to map the right half of the $Z_{e}$ plane to the inside of a unit disk in the $\Gamma$ plane. However, this method still requires to pick a large number of points in the 
unit disk in the $\Gamma$ plane, test (28), and then repeat this process for a large number of frequencies $\omega$ before one can reasonably be sure that Llewellyn's conditions are met for a large set of points in the right half of the $Z_{e}$ plane and for a large set of frequencies.

\subsubsection{Direct Stability Analysis of the Three-port Net- work}

By using the proposed stability criterion in Theorem 1 , it is possible to design the dual-user teleoperation system controller quickly and without a need to iteratively and numerically test a number of conditions across the load impedance space and the frequency range. Let us take the same choices of $Z_{m 1}, Z_{m 2}, Z_{s}$, $C_{m 1}, C_{m 2}$ and $C_{s}$ as in subsection 4.2.1. In this case, it can be shown that the symmetrization condition (16) will hold only if $\alpha=\frac{1}{2}$, for which we find the equivalent reciprocal three-port network and replace $s=j \omega$. It is easy to see that the stability (weak stability) conditions (17a)-(17f) turn out to be

$$
\begin{aligned}
& K_{v m 1}>0 \quad(\geq 0) \\
& K_{v m 2}>0 \quad(\geq 0) \\
& K_{v s}>0 \quad(\geq 0) \\
& \frac{7}{8} K_{v m 1} K_{v m 2}+\frac{1}{8 \omega^{2}} K_{p m 1} K_{p m 2}-\frac{Q_{m 1} Q_{m 2}}{8 \omega^{2}}>0 \\
& \frac{7}{8} K_{v m 1} K_{v s}+\frac{1}{8 \omega^{2}} K_{p m 1} K_{p s}-\frac{Q_{m 1} Q_{s}}{8 \omega^{2}}>0 \quad(\geq 0) \\
& \frac{7}{8} K_{v s} K_{v m 2}+\frac{1}{8 \omega^{2}} K_{p s} K_{p m 2}-\frac{Q_{s} Q_{m 2}}{8 \omega^{2}}>0 \quad(\geq 0)
\end{aligned}
$$

where $Q_{m 1}=\sqrt{K_{v m 1}^{2} \omega^{2}+K_{p m 1}^{2}}, Q_{m 2}=$ $\sqrt{K_{v m 2}^{2} \omega^{2}+K_{p m 2}^{2}}$, and $Q_{s}=\sqrt{K_{v s}^{2} \omega^{2}+K_{p s}^{2}}$. Now, under (29) and (30), condition (32)-(34) will be fulfilled for all frequencies $\omega$ if the gains of the PD controllers $C_{m 1}, C_{m 2}$, and $C_{s}$ satisfy

$$
\frac{K_{v m 1}}{K_{p m 1}}=\frac{K_{v m 2}}{K_{p m 2}}, 7-4 \sqrt{3} \leq \frac{K_{v m 1} K_{p s}}{K_{p m 1} K_{v s}} \leq 7+4 \sqrt{3}
$$

On the other hand, under (35), condition (17g) becomes

$$
5 K_{v m 1} K_{v s}+\frac{K_{p m 1} K_{p s}}{\omega^{2}}-\frac{Q_{m 1} Q_{s}}{\omega^{2}}>0 \quad(\geq 0)
$$

One can see that condition (36) will be fulfilled for all frequencies $\omega$ if the gains of the PD controllers $C_{m 1}$ and $C_{s}$ satisfy

$$
5-2 \sqrt{6} \leq \frac{K_{v m 1} K_{p s}}{K_{p m 1} K_{v s}} \leq 5+2 \sqrt{6}
$$

So, a sufficient, frequency-independent, and compact condition for stability of the above-described positionposition dual-user teleoperation systems is

$\frac{K_{v m 1}}{K_{p m 1}}=\frac{K_{v m 2}}{K_{p m 2}}, 5-2 \sqrt{6} \leq \frac{K_{v m 1} K_{p s}}{K_{p m 1} K_{v s}} \leq 5+2 \sqrt{6}$ where all control gains are nonnegative (note that the ratios in (38) are merely artifacts of our presentation of the stability conditions meaning that division by zero can be avoided).

At the first glance, the constraint $\alpha=\frac{1}{2}$ imposed by the symmetrization condition (16) seems very limiting. However, one must note that various combinations of authority sharing and teleoperation control laws exist and $\alpha=\frac{1}{2}$ is only an artifact of using CLC authority sharing laws in conjunction with position-position teleoperation control laws. For instance, by changing the authority sharing laws (24) to the masters-correspondence-withenvironment-transfer (MCET) law proposed in [21], for the same dynamics for the master and the slave and the same position-position control laws as in Section IV.A, the symmetrization condition (16) holds for any $\alpha$ because $Z_{13} Z_{21} Z_{32}-Z_{12} Z_{23} Z_{31}$ is identical to zero.

\subsection{Force-position Dual-user Teleoperation}

Force-position control is another special case of fourchannel control that requires a force sensor to measure the interactions between the slave and its environment. In this control architecture, we have $C_{m 1}=$ $C_{m 2}=C_{3}=C_{4 m 1}=C_{4 m 2}=C_{5}=C_{6 m 1}=C_{6 m 2}=0$. Also, for good position and force tracking, we need $C_{1}=C_{s}$ and $C_{2 m 1}=C_{2 m 2}=1$, respectively. Then, the impedance matrix of dual-user teleoperation system can be found from (25) but is not shown here.

In this case, the symmetrization condition (16) is met only if $Z_{m 1}=Z_{m 2}$. Take $Z_{m 1}=Z_{m 2}=M_{m} s$, $Z_{s}=M_{s} s$, and the controller $C_{s}=\frac{K_{p s}+K_{v s} s}{s}$. With $s=j \omega$, the stability (weak stability) conditions (17a)(17c) and (17g) turn out to be

$$
\begin{aligned}
& -\alpha K_{v s}>0 \quad(\geq 0) \\
& (\alpha-1) K_{v s}>0 \quad(\geq 0) \\
& K_{v s}>0 \quad(\geq 0) \\
& 2 \alpha^{2}(1-\alpha) K_{v s}\left(\sqrt{K_{v s}^{2}+\left(\frac{K_{p s}}{\omega}-M_{s} \omega\right)^{2}} \sqrt{K_{v s}^{2}+\frac{K_{p s}^{2}}{\omega^{2}}}\right. \\
& \left.-K_{v s}^{2}+\frac{K_{p s}^{2}}{\omega^{2}}-K_{p s} M_{s}\right)>0 \quad(\geq 0)
\end{aligned}
$$

Also, the left side of (17d)-(17f) becomes identical to zero. Since $0 \leq \alpha \leq 1$, the inequality conditions (39)(42) will be fulfilled as equalities if and only if

$$
K_{v s}=0
$$

which corresponds to a weakly stable system for any $\alpha$ in the prescribed range. Again, at the first glance, the constraint $K_{v s}=0$ seems limiting. However, this condition is obtained even when using Llewellyn's criterion for bilateral teleoperation systems if force-position teleoperation control laws are employed [22]. Interestingly, the force-position controller can be modified to allow for a nonzero 


\section{TABLE 1}

The controllers gains used in $(A)$ simulations of the position-position system, (B) simulations of the force-position system, and (C) experiments of the position-position system.

\begin{tabular}{l|ll|ll|ll}
\hline & \multicolumn{2}{|c|}{ Master \#1 } & \multicolumn{2}{c|}{ Master \#2 } & \multicolumn{2}{c}{ Slave } \\
\hline (A) & $K_{p m 1}$ & 630 & $K_{p m 2}$ & 630 & $K_{p s}$ & 25200 \\
& $K_{v m 1}$ & 29.4 or 60 & $K_{v m 2}$ & 29.4 & $K_{v s}$ & 1176 \\
\hline (B) & $K_{p m 1}$ & - & $K_{p m 2}$ & - & $K_{p s}$ & 13 \\
& $K_{v m 1}$ & - & $K_{v m 2}$ & - & $K_{v s}$ & 0 or 15 \\
\hline (C) & $K_{p m 1}$ & 600 & $K_{p m 2}$ & 800 & $K_{p s}$ & 1200 \\
& $K_{v m 1}$ & 300 & $K_{v m 2}$ & 400 & $K_{v s}$ & 600 \\
\hline
\end{tabular}

derivative term while maintaining absolute stability. In fact, if the proportional-derivative (PD) controller $F_{c s}=\frac{K_{p s}}{s}\left(V_{h}-V_{e}\right)+K_{v s}\left(V_{h}-V_{e}\right)$ for the slave is modified to the proportional-plus-damping (P+D) controller $F_{c s}=\frac{K_{p s}}{s}\left(V_{h}-V_{e}\right)-K_{v s} V_{e}$ [23], then $K_{v s}=0$ is no longer required for absolute stability. Intuitively, choices made with respect to the teleoperation control laws (e.g., position-position versus forceposition), authority sharing laws (e.g., CLC versus MCET) and specific controller choices (e.g., PD versus $\mathrm{P}+\mathrm{D}$ ) influence the absolute stability and the proposed criterion provides a systematic way to study this.

\section{SimULATIONS AND EXPERIMENTS}

In this section, the stability condition has been applied to assess the stability of the case study trilateral haptic systems described in Section IV. For brevity, we do not report here the experiment results of a the exercise for the force-position system. For checking the stability of the position-position and force-position dual-user teleoperation systems, the master \#2 and the slave were connected to passive terminations while the input energy at the master \#1's port (i.e., the energy dissipation in the three-port network terminated in ports 2 and 3) was measured. According to (5), the system is stable (weakly stable) if and only if, at all times $t>0$, we have

$$
E(t)=\int_{0}^{t} f_{h 1}(\tau) V_{h 1}(\tau) \mathrm{d} \tau>0 \quad(\geq 0)
$$

\subsection{Simulations}

The position-position and force-position systems have been simulated in MATLAB/Simulink. There is no time delay in the communication channel between the masters and the slave. Three 1-DOF robots as the two masters and the slave are modeled by masses $M_{m 1}=0.7, M_{m 2}=0.7$, and $M_{s}=0.5$, respectively. The master \#2 and the slave are connected to passive LTI terminations with transfer functions $\frac{1}{s+1}$, which are strictly passive as, for $s=j \omega$, we have $\operatorname{Re}\left(\frac{1}{\mathrm{~s}+1}\right)=$ $\frac{1}{\omega^{2}+1}>0$ when $\omega>0$. A sine-wave input $F_{h 1}$ is applied to the master \#1's port.

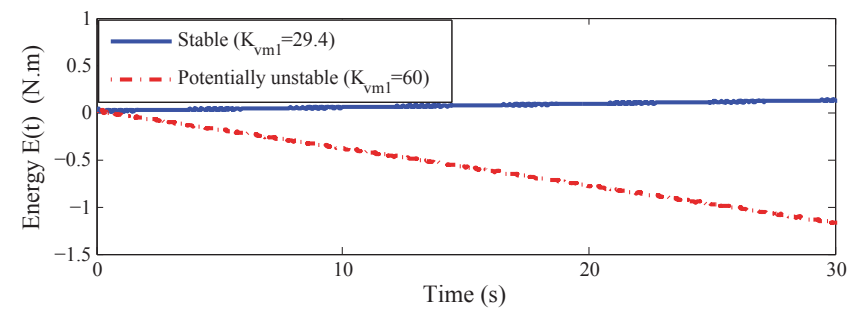

Fig. 2. Input energy at the master \#1's port of a position-position dual-user teleoperation system. Simulation parameters are listed in Table 1(A) for the stable case with $K_{v m 1}=29.4$, and for the potentially unstable case with $K_{v m 1}=60$.

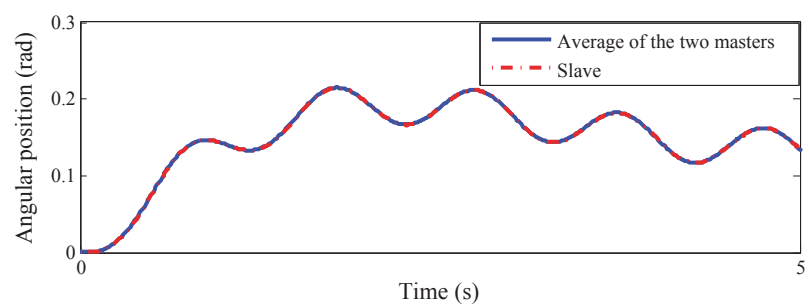

Fig. 3. Simulation results for the dual-user teleoperation system. The desired and actual positions for the slave are shown. A sinusoidal force was applied to the master \#1 while the master \#2 and the slave were connected to passive terminations. Simulation parameters are listed in Table $1(\mathrm{~A})$ for the stable case with $K_{v m 1}=29.4$.

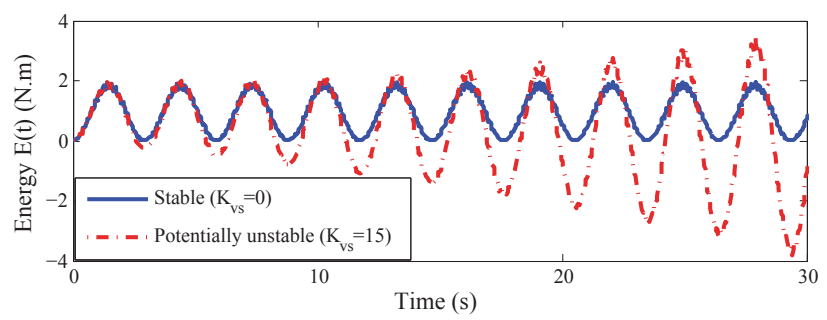

Fig. 4. Input energy at the master \#1's port of a forceposition dual-user teleoperation system. Simulation parameters are listed in Table $1(\mathrm{~B})$ for the stable case with $K_{v s}=0$, and for the potentially unstable case with $K_{v s}=15$.

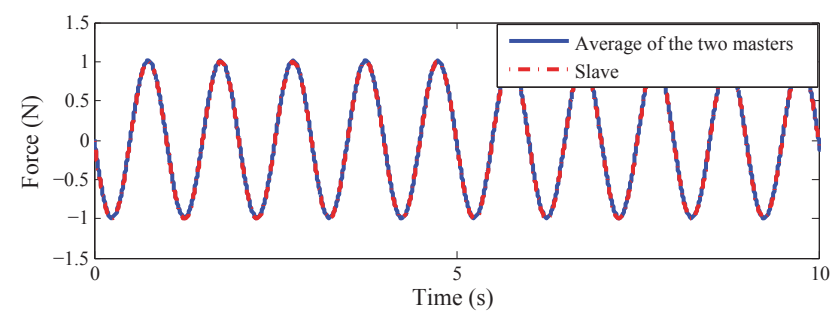

Fig. 5. Simulation results for the dual-user teleoperation system. The desired and actual forcrs for the slave are shown. A sinusoidal force was applied to the master \#1 while the master \#2 and the slave were connected to passive terminations. Simulation parameters are listed in Table 1(B) for the stable case with $K_{v s}=0$. 


\subsubsection{Position-position trilateral teleoperation system}

According to (38), the stability of the position-position dual-user teleoperation system should depend on the controllers gains. In the simulations, the controllers gains $K_{p m 1}, K_{v m 1}, K_{p m 2}, K_{v m 2}, K_{p s}$, and $K_{v s}$ were chosen according to Table 1(A). Also, $\alpha=\frac{1}{2}$. The input energy (44) profiles are plotted in Figure 2. As it can be seen, if the controllers gains are selected according to (38), i.e., as listed in Table 1(A) with $K_{v m 1}=29.4$, then the input energy at port 1 is always positive at all times, indicating stability of the trilateral haptic system. However, when we change $K_{v m 1}$ to 60 , which violates (38), the input energy will become negative at least for a period of time, indicating potential instability of the trilateral system. We get similar results if we repeat the above simulations after replacing the strictly passive terminations $\frac{1}{s+1}$ by the passive terminations $\frac{1}{s}$, and do not report its results for brevity. For the case of $k_{v m 1}=29.4$, Figure 3 depicts the average positions of two masters versus the slave position. These results agree with the stability condition (38).

\subsubsection{Force-position trilateral teleoperation system}

According to (43), the controllers gains $K_{p m 1}, K_{v m 1}$, $K_{p m 2}, K_{v m 2}, K_{p s}$, and $K_{v s}$ of the force-position dualuser teleoperation system were chosen as shown in Table 1(B). Similar to the position-position case, we choose $\alpha=\frac{1}{2}$. Note that in the force-position scheme, there is no local position controller for either of the master robots. According to (43), the stability of the force-position trilateral teleoperation system is guaranteed if $K_{v s}=0$. The input energy (44) profiles are plotted in Figure 4. As it can be seen, if the controllers gains are selected according to (43), i.e., as listed in Table 1 (B) with $K_{v s}=0$, then the input energy at port 1 is always positive at all times, indicating stability of the trilateral haptic system. However, when we change $K_{v s}$ to 15 , which violates (43), the input energy will become negative at least for a period of time, indicating potential instability of the trilateral system. We get similar results if we repeat the above simulations after replacing the strictly passive terminations $\frac{1}{s+1}$ by the passive terminations $\frac{1}{s}$, and do not report its results for brevity. For the case of $K_{v s}=0$, Figure 5 depicts the average forces of two masters versus the slave force. These results agree with the stability condition (43).

\subsection{Experiments}

We use a dual-user teleoperation system comprising two Phantom Premium 1.5A robots (Sensable Technologies/Geomagic, Wilmington, MA) as the master $\# 1$ and as the master \#2, and a Phantom Omni robot as the slave. Out of the three actuated joints of each robot, the first joint, which rotates about the vertical, is considered in the experiments while the second and

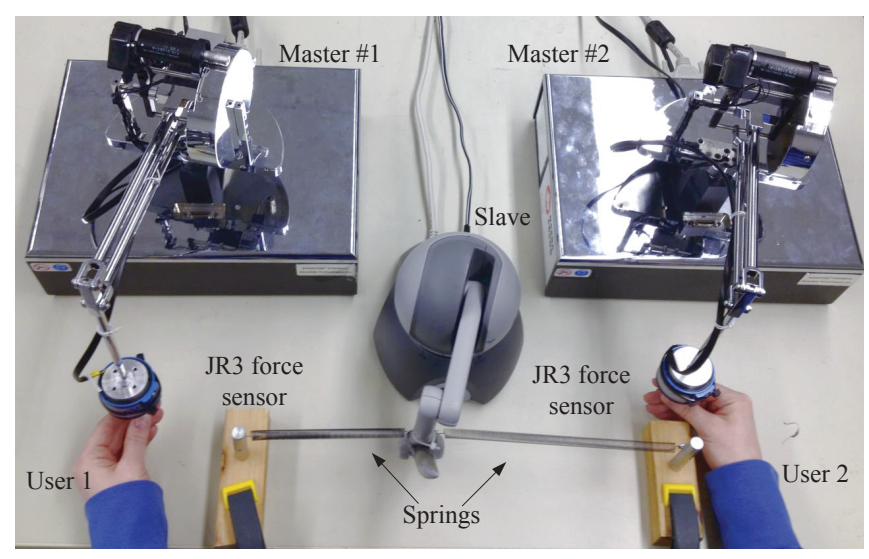

Fig. 6. Experimental setup where the master \#1 and the master \#2 are controlled by human users. The above shows the case where the slave is connected via passive spring to stiff wall. Other 2 cases are set up in a similar way.

the third joints, which form a parallel mechanism, are locked using high-gain controllers. The master \#1 and the master \#2 are equipped with JR3 6-DOF force (JR3, Inc., Woodland, CA) for measuring the applied forces.

The experimental setup is shown in Figure 6, where two human users interacts with the master \#1's and the master \#2 while the slave is in free motion, physically connected via a passive spring to a stiff wall, or physically clamped to a stiff wall. The positionposition controllers gains are chosen according to Table $1(\mathrm{C})$, meeting the theoretical stability condition (38). The input energy (44) profiles are plotted in Figure 7 when the slave is connected to different passive terminations. As it can be seen, for all 3 cases, the input energies at the master \#1's port and at the master \#2's port are always positive, which means the trilateral haptic system is stable. For the case in which the slave is physically connected via a passive spring to a stiff wall, Figure 8 depicts the average positions of two masters versus the slave position. This time profile of positions further corroborates the stability of the system. These experimental results agree with the stability condition (38).

\section{Conclusions And Future Works}

We presented a closed-form stability criterion for a three-port network based on its impedance (admittance) matrix. While the proposed criterion (Theorem 1) can be used for stability analysis of a general class of three-port networks in a variety of applications, we elaborated on its application in stability analysis of trilateral haptic systems. Through simulations and experiments involving dual-user haptic teleoperation of one slave robot, the proposed stability criterion was validated. While equations (17a)-(17g) give necessary and sufficient conditions for a trilateral haptic system's absolute stability, the symmetrization condition 


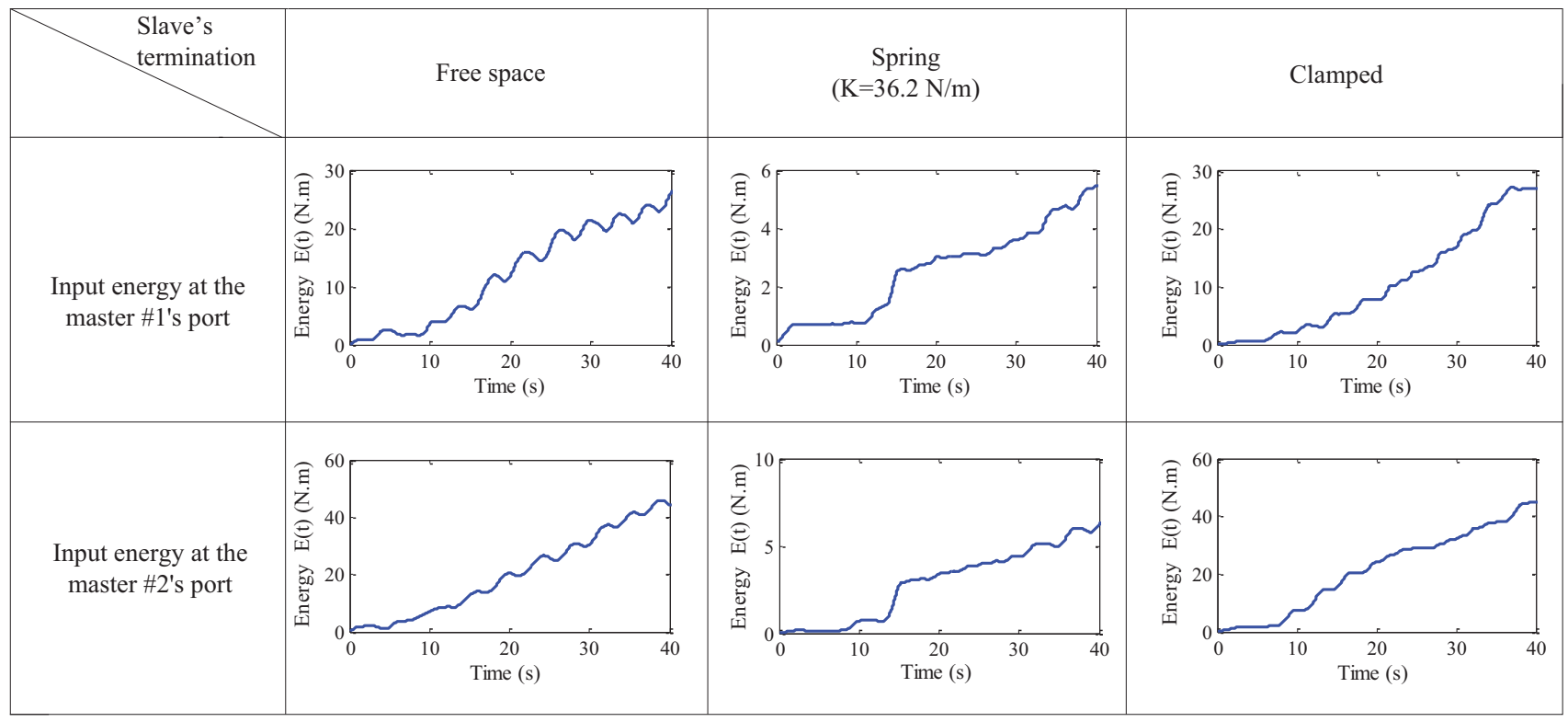

Fig. 7. Input energy $E_{t}$ at the master \#1's port and at the master \#2's port of a position-position dual-user teleoperation system. Experimental parameters are listed in Table 1(C). The master \#1 and the master \#2 are held by users. The slave is either in free motion, physically connected via a passive spring to a stiff wall, or physically clamped.

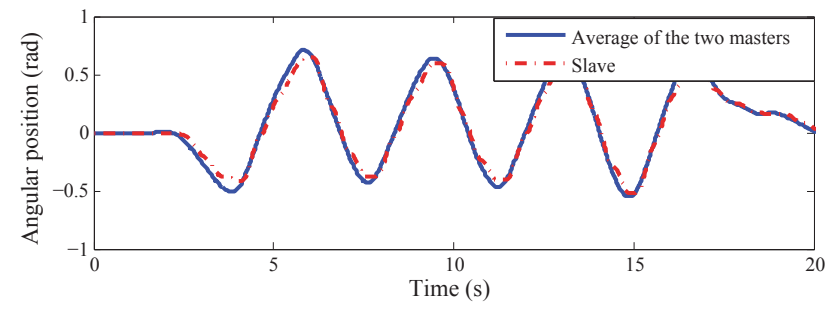

Fig. 8. Experimental results for the dual-user teleoperation system. The desired and actual positions for the slave are shown. The master robot \#1 is moved by the human operator and the master \#2 and the slave are Physically connected via a passive spring to a stiff wall. Parameters are listed in Table 1(C).

is a limiting factor. However, it is mild and mostly fulfilled by appropriate choice of free parameters in the three-port network including the teleoperation control structure, authority sharing laws, and control gains. The symmetrization condition involves the actual values of the teleoperator model parameters. The robustness of the symmetrization condition against variations in these parameters varies from case to case and needs to be investigated before proceeding to checking the stability conditions (17a)-(17g). In the future, the proposed stability criterion can be used to investigate the stability of trilateral haptic systems that experience time delays in their communication channels. Extending the proposed stability criterion to the case of multi-DOF trilateral haptic systems requires further investigation as well. Also, while we have focused on the stability analysis, future work can investigate the stability-transparency trade-offs for trilateral haptic systems in light of the proposed stability criterion.

\section{ACKNOWLEDGMENTS}

The authors thank Ali Jazayeri for helping with the experimental setup. This research was supported by the Natural Sciences and Engineering Research Council (NSERC) of Canada, by the Natural Science Foundation of China (NFSC, Grant No. 51277022), and by the China Scholarship Council (CSC) under grant [2011]3005.

\section{References}

[1] B. Hannaford, "A design framework for teleoperators with kinesthetic feedback," IEEE Transactions on Robotics and Automation, vol. 5, pp. 426-434, 1989.

[2] F. Llewellyn, "Some fundamental properties of transmission systems," Proceedings of the IRE, vol. 2, no. 1, pp. 271-283, 1952.

[3] W. Ku, "A simple dervation for the stability criterion of linear active two-port," Proceedings of the IEEE, pp. 310 - 311, March 1965.

[4] S. Haykin, Active Network Theory. Reading, MA: AddisonWesley, 1970.

[5] C. R. Carignan and P. A. Olsson, "Cooperative control of virtual objects over the internet using force-reflecting master arms," in Proc. of IEEE Int. Conf. on Rob. And Auto., vol. 2, pp. 1221-1226, 2004.

[6] S. Nudehi, R. Mukherjee, and M. Ghodoussi, "A sharedcontrol approach to haptic interface design for minimally invasive telesurgical training," IEEE Transactions on Control Systems Technology, vol. 13, no. 4, pp. 588-592, July 2005.

[7] W. T. Lo, Y. Liu, I. Elhajj, N. Xi, Y. Wang, and T. Fukuda, "Cooperative teleoperation of a multirobot system with force reflection via internet," IEEE/ASME Transactions on Mechatronics, vol. 9, no. 4, pp. 661 -670, December 2004. 
[8] R. F. Kuo and T. H. Chu, "Unconditional stability boundaries of a three-port network," IEEE Transactions on Microwave Theory and Techniques, vol. 58, no. 2, pp. 363-371, December 2010.

[9] E. Tan, "Simplified graphical analysis of linear three-port stability," IEE Proceedings on Microwaves, Antennas and Propagation, vol. 152, no. 4, pp. 209-213, August 2005.

[10] B. Khademian and K. Hashtrudi-Zaad, "Shared control architectures for haptic training: Performance and coupled stability analysis," The International Journal of Robotics Research, vol. 30, pp. 1627-1642, 2011.

[11] J. Boehm and W. Albright, "Unconditional stability of a threeport network characterized with s-parameters," IEEE Transactions on Microwave Theory and Techniques, vol. 35, no. 6, pp. 582 - 586, January 1987.

[12] J. Choma and W. K. Chen, Feedback Networks: Theory and Circuit Applications. World Scientific Publishing Company, 2007.

[13] W. Ku, "Stability of linear active nonreciprocal n-ports," J. Franklin Inst., no. 276, pp. 207 - 224, 1963.

[14] V. Mendez and M. Tavakoli, "A passivity criterion for n-port multilateral haptic systems," in 2010 49th IEEE Conference on Decision and Control (CDC), December 2010, pp. 274- 279.

[15] D. Youla, "A stability characterization of the reciprocal linear passive n-port," Proc. IRE, vol. 47, pp. 1150-1151, 1959.

[16] S. Boyd and O. Chua, "On the passivity criterion for LTI nport," Circuit theory and applications, vol. 10, pp. 323-333, 1982.

[17] H. J. Marquez, Nonlinear Control Systems Analysis and Design. Wiley, 2003.

[18] D. Youla, "A note on the stability of linear, nonreciprocal nport," Proc. IRE, vol. 48, pp. 121-122, 1960.

[19] H. Anton and C. Rorres, Elementray linear algebra: Applications version. Wiley, 2005, vol. 9.

[20] C. M. Gewertz, Network Synthesis: Synthesis of a Finite FourTerminal Network from Its Prescribed Driving-Point Functions and Transfer Function. The Williams and Wilkins Company, 1933.

[21] B. Khademian and K. Hashtrudi-Zaad, "Dual-user teleoperation systems: New multilateral shared control architecture and kinesthetic performance measures," IEEE/ASME Transactions on Mechatronics, vol. 17, no. 5, pp. 895-906, October 2012.

[22] M. Tavakoli, A. Aziminejad, R. Patel, and M. Moallem, "Highfidelity bilateral teleoperation systems and the effect of multimodal haptics," IEEE Transactions on Systems, Man, and Cybernetics, Part B: Cybernetics, vol. 37, no. 6, pp. $1512-1528$, December 2007.

[23] B. Willaert, B. Corteville, D. Reynaerts, H. V. Brussel, and E. B. V. Poorten, "A mechatronic analysis of the classical position-force controller based on bounded environment passivity," The International Journal of Robotics Research, vol. 30, pp. 444-462, 2011.

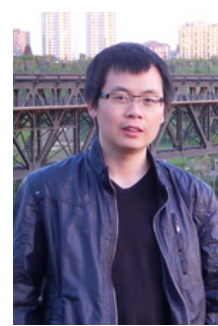

Jian Li received his B.Sc. and M.Sc. degrees in Detection Technology and Automatic Equipment from the University of Electronic Science and Technology of China, Sichuan, China in 2007 and 2010. Currently, he is a Ph.D. student in the School of Energy Science and Engineering, University of Electronic Science and Technology of China and he has been awarded a scholarship under the State Scholarship Fund to pursue his study in the University of Alberta as a Visiting Doctoral student. His main research interests include haptics and teleoperation control, optimal control, and power system.

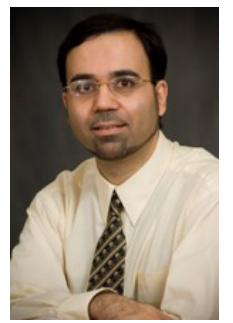

Mahdi Tavakoli received his BSc and MSc degrees in Electrical Engineering from Ferdowsi University and K.N. Toosi University, Iran, in 1996 and 1999, respectively. He then received his $\mathrm{PhD}$ degree in Electrical and Computer Engineering from the University of Western Ontario, London, ON, Canada, in 2005. In 2006, he was a post-doctoral research associate at Canadian Surgical Technologies and Advanced Robotics (CSTAR), London, ON, Canada. In 2007-2008, and prior to joining the Department of Electrical and Computer Engineering at the University of Alberta, Dr. Tavakoli was an NSERC PostDoctoral Fellow with the BioRobotics Laboratory of the School of Engineering and Applied Sciences at Harvard University, Cambridge, MA, USA. Dr. Tavakolis research interests broadly involve the areas of robotics and systems control. Specifically, his research focuses on haptics and teleoperation control, medical robotics, and imageguided surgery. Dr. Tavakoli is the first author of the book Haptics for Teleoperated Surgical Robotic Systems (World Scientific, 2008).

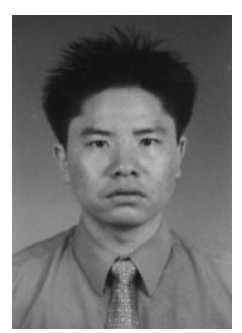

Qi Huang (StM99, M03, SM09) received his BS degree in Electrical Engineering from Fuzhou University in 1996, MS degree from Tsinghua University in 1999, and Ph.D degree from Arizona State University in 2003. $\mathrm{He}$ is currently a professor at University of Electronic Science and Technology of China (UESTC) and the Deputy Dean of School of Energy Science and Engineering, UESTC, and the Director of Sichuan State Provincial Lab of Power System Wide-area Measurement and Control. His current research and academic interests include power system high performance computing, power system instrumentation, power system monitoring and control, and integration of distributed generation into the existing power system infrastructure. 